\title{
$\mathrm{DNP}$ 제어기에 의한 비선형 동적 매니퓰레이터의 실시간 경로 제어
}

\author{
조현섭 $1^{*}$ \\ ${ }^{1}$ 청운대학교 디지털방송공학과

\section{Real-Time Collision-Free Trajectory Contol of Nonlinear Dynamic Manipulator Control Using DNP Controller}

\author{
Hyun-Seob Cho ${ }^{1^{*}}$ \\ ${ }^{1}$ Dept. of Digital Broadcasting \& Electronic Engineering, Chungwoon Univ.,
}

\begin{abstract}
요 약 본 논문은 가변 구조 제어기의 단점인 도달영역에서의 파리미터의 불확실성과 외부 외란에 대한 민감성을 감소시키는 방안으로 DNP제어를 제시한다. 비선형 동적 매니퓰레이터를 통하여 시스템의 상태 궤적이 초기 위치에 서부터 평형점에 이르기까지 외란과 파라미터의 불확실성에 강인하게 되며 아울러 목표 직각 좌표까지 도달시간 뿐 만 아니라 평형점까지의 도달시간도 감소하게 되는 특성을 보이고자 한다. 제안된 제어 구조의 효과는 시뮬레이션을 통해 증명하였다.
\end{abstract}

\begin{abstract}
This study develops a variable structure controller using nonlinear surface instead of the fixed, which has been the robustness against parameter variations and extraneous disturbance during the reaching phase. reaching time of the system trajectory is faster than the fixed method. This proposed scheme has better performance than the conventional method in reaching time, parameter variation and extraneous disturbance. The effectiveness of the proposed control scheme is verified by simulation results.
\end{abstract}

Key Words : Dynamic Neural Processor, Association, Plants of Auto-Equipment Systems, Robustness Control, Nonlinear Systems

\section{1. 서론}

신경망 분야의 발전으로 인해 자동화 시스템의 제어에 새로운 방향이 제시되고 있으며 신경망은 함수 사상과 동적 반복 능력이라는 장점에 의해 좌표 변환 학습에 사 용될 수 있다[1-3]. 본 논문에서 논의된 신경망 구조는 임 의 신경활동이 여기와 금지의 신경 생리학적인 근거에 기초하여 개발된 dynamic neural processor (DNP)이다. 또한 신경 집단의 최소 위상은 여기(양성), 금지(음성), 여기-금지(여기에서 금지로의 시냅스 연결), 금지-여기(금 지에서 여기로의 시냅스 연결)궤환 루프를 포함한다. 신 경 집단의 가장 중요한 속성의 하나는 비선형 시스템 해 석의 관점에서 기술할 수 있는 몇 가지 기본적인 속성이
다. 예를 들어 이들 패턴은 작업 공간 좌표와 모형 다리 의 대응하는 관절각을 나타낼 수 있다. 이들 두 패턴 집 합의 연상은 기본적으로 자동화 시스템의 로봇 계열에서 는 역기구학적으로 계산된다. 이러한 전제를 바탕으로 자 동화 시스템에서 매니퓰레이터 제어 문제의 응용에 DNP 를 제안하였다. 따라서 $\mathrm{DNP}$ 의 구조에 대해 설명하고 신 경망의 적응적인 가중치에 대한 학습 알고리듬을 제시한 다. 제안된 제어 기법의 효과는 시뮬레이션에 의하여 확 인하였다.

\section{2. 가변 구조 제어이론}

*교신저자: 조현섭(chohs@chungwoon.ac.kr)

접수일 09년 12월 22일 수정일 10년 02월 17일
게재확정일 10 년 03 월 18 일 


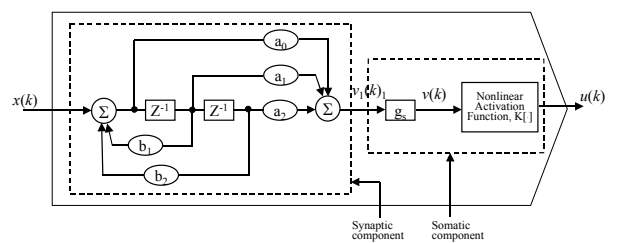

[그림 1] Synaptic \& Somatic 요소로 구성되는 DNU구조

적응적인 시냅스 강도를 갖는 $\mathrm{DNP}$ 의 기본적인 기능 은 DNU에 기초한다[4-7]. DNU는 기억 요소와 그림 1과 같이 feedforward 및 feedback 시냅스 가중치로 구성된다. 이 동적 구조의 출력은 시변 비선형 활성화 함수에 대한 인자를 구성한다.DNU는 크게 (i)synaptic operation과 (ii)somatic operation의 두 가지의 동작을 수행한다. 전자 는 feedforward 및 feedback 시냅스 가중치의 적응 (adaptation)에 대응되고 후자는 비선형 활성화 함수의 이 득(형태)의 적응에 대응된다. DNU는 각각 비선형 활성화 함수에 이어지는 2 차 구조를 나타내는 시냅스 가중치 $\mathrm{aff}_{\mathrm{ff}}$ 와 $\mathrm{b}_{\mathfrak{f b}}$ 에 의해 가중되는 feedforward 및 feedback 경로인 지연 요소로 구성된다.

$$
\begin{array}{r}
\mathrm{v}_{1}(\mathrm{k})=-\mathrm{b}_{1} \mathrm{v}_{1}(\mathrm{k}-1)-\mathrm{b}_{2} \mathrm{v}_{1}(\mathrm{k}-2) \\
+a_{0} \mathrm{~s}(\mathrm{k})+\mathrm{a}_{1} \mathrm{~s}(\mathrm{k}-1)+\mathrm{a}_{2} \mathrm{~s}(\mathrm{k}-2)
\end{array}
$$

이때 $\mathrm{s}(\mathrm{k}) \in \mathrm{R}^{\mathrm{n}}$ 은 신경 입력 벡터이고, $\mathrm{v}_{1}(\mathrm{k}) \in \mathrm{R}^{1}$ 은 동 적 구조의 출력이며, $\mathrm{u}(\mathrm{k}) \in \mathrm{R}^{1}$ 은 신경 출력, $\mathrm{k}$ 는 이산 시 간 지표, $z^{-1}$ 은 단위 지연 연산자, $\mathrm{a}_{\mathrm{ff}}=\left[\mathrm{a}_{0}, \mathrm{a}_{1}, \mathrm{a}_{2}\right]$ 와 $\mathrm{b}_{\mathrm{fb}}=\left[\mathrm{b}_{1}\right.$, $\mathrm{b}_{2}$ ]는 다음과 같이 정의된다.

$\Gamma^{\mathrm{T}}\left(\mathrm{k}, \mathrm{v}_{1}, \mathrm{~s}\right)$

$=\left[\mathrm{v}_{1}(\mathrm{k}-1) \mathrm{v}_{1}(\mathrm{k}-2) \mathrm{s}(\mathrm{k}) \mathrm{s}(\mathrm{k}-1) \mathrm{s}(\mathrm{k}-2)\right]$

$\zeta^{\mathrm{T}}\left(\mathrm{a}_{\mathrm{ff}}, \mathrm{b}_{\mathrm{fb}}\right)=\left[-\mathrm{b}_{1}-\mathrm{b}_{2} \mathrm{a}_{0} \mathrm{a}_{1} \mathrm{a}_{2}\right]$

( $\mathrm{T}:$ trans pose $)$

(2)와 (3)에 의해 식 (1)은 다음과 같이 된다.

$\mathrm{v}_{1}(\mathrm{k})=\Gamma(\mathrm{k}, \mathrm{v}-1, \mathrm{~s}) \mathrm{\zeta}^{\mathrm{T}}\left(\mathrm{a}_{\mathrm{ff}}, \mathrm{b}_{\mathrm{fb}}\right)$

$\mathrm{v}_{1}(\mathrm{k})$ 에 대한 비선형 사상은 다음과 같은 출력을 낸다.

$\mathrm{u}(\mathrm{k})=\Psi\left[\mathrm{g}_{\mathrm{s}} \mathrm{v}_{1}(\mathrm{k})-\theta\right]$
이때 $\Psi[$.$] 는 비선형 활성화 함수로서 보통은$ sigmoidal 함수이고 $\mathrm{g}_{\mathrm{s}}$ 는 활성화 함수의 기울기를 제어하 는 somatic gain이며, $\theta$ 는 뉴런을 점화시키기 위한 임계 치(threshold)이다. 여기와 금지 입력 모두에 대한 수학적 인 동작을 확장하기 위해 [-1,1]에 대한 활성화 함수를 다 음과 같이 정의한다.

$\Psi[\mathrm{v}(\mathrm{k})]=\tanh \left[g_{s} v_{1}(k)-\theta\right]=\tanh [v(k)]$

이때 $\mathrm{v}(\mathrm{k})=\mathrm{g}_{\mathrm{s}} \mathrm{v}_{1}(\mathrm{k})-\ominus$ 이다.

\section{Dynamic Neural Processor(DNP)}

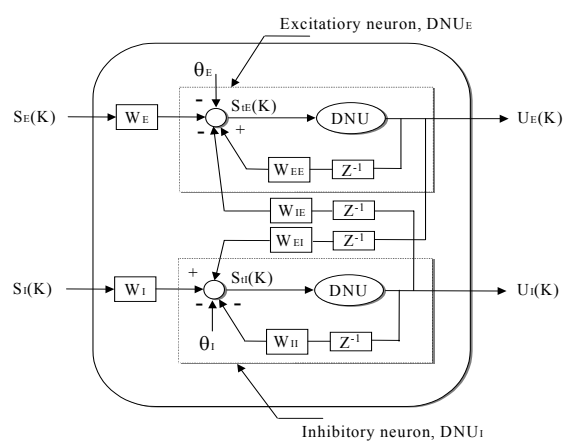

[그림 2] DNP의 구조

$\mathrm{DNP}$ 는 그림 2에서와 같이 여기와 금지 방식으로 결합 된 두개의 DNU로 구성된다. 이 구조에서, $\mathrm{s}_{\lambda}(\mathrm{k})$ 와 $\mathrm{u}_{\lambda}(\mathrm{k})$ 는 첨자 $\lambda$ 가 여기 $\mathrm{E}$ 나 금지 I 상태를 가리킬 때 신경 계 산 단위의 자극(입력)과 상태 반응(출력)을 나타낸다. $\mathrm{s}_{\mathrm{t} \lambda}$ (k)는 신경 단위의 총 입력을 나타내고 $\mathrm{W}_{\lambda \lambda}$ 는 자기 시냅 스 연결 강도(그림 2 의 $\mathrm{w}_{\mathrm{EE}}, \mathrm{w}_{\mathrm{II}}$ )를 나타내고 $\mathrm{w}_{\lambda \lambda}$ '는 한 뉴런에서 다른 뉴런으로의 상호 시냅스 연결 강도(그림 2 의 $\left.\mathrm{W}_{\mathrm{IE}}, \mathrm{W}_{\mathrm{EI}}\right)$ 를 나타낸다. 신경 계산 단위인 $\mathrm{DNU}$ 에 의해 여기되는 기능적 동역학은 식(1)에 나타낸 바와 같이 2차 차분 방정식으로 정의된다. 제안된 신경 프로세서의 여기 및 금지 신경 단위에 의해 시간 $(\mathrm{k}+1)$ 에 발생되는 상태 변수 $\mathrm{u}_{\mathrm{E}}(\mathrm{k}+1)$ 와 $\mathrm{u}_{\mathrm{I}}(\mathrm{k}+1)$ 는 다음과 같이 모델링된다.

$$
\begin{aligned}
& \mathrm{u}_{\mathrm{E}}(\mathrm{k}+1)=\mathrm{E}\left[\mathrm{u}_{\mathrm{E}}(\mathrm{k}), \mathrm{v}_{\mathrm{E}}(\mathrm{k})\right] \\
& \quad \text { and } \mathrm{u}_{\mathrm{I}}(\mathrm{k}+1)=\mathrm{I}\left[\mathrm{u}_{\mathrm{I}}(\mathrm{k}), \mathrm{v}_{\mathrm{I}}(\mathrm{k})\right]
\end{aligned}
$$

이때 $\mathrm{V}_{\mathrm{E}}(\mathrm{k})$ 와 $\mathrm{V}_{\mathrm{I}}(\mathrm{k})$ 는 내부 임계치보다 더 큰 입력을 받 
아들이는 신경단위에서 뉴런의 비를 나타내고 $\mathrm{E}$ 와 I는 뉴런의 여기와 금지 작용을 나타낸다. 임계치 보다 더 큰 입력을 받아들이는 뉴런은 비선형 함수 $\mathrm{v}_{\lambda}(\mathrm{k})$ 로 주어진 다. 여기와 금지 신경 단위에 수반되는 총 입력은 각각 다음과 같다.

$\mathrm{s}_{\mathrm{uE}}(\mathrm{k})=\mathrm{w}_{\mathrm{E}} \mathrm{s}_{\mathrm{E}}(\mathrm{k})+\mathrm{w}_{\mathrm{EE}} \mathrm{u}_{\mathrm{E}}(\mathrm{k}-1)-\mathrm{w}_{\mathrm{EE}} \mathrm{u}_{\mathrm{I}}(\mathrm{k}-1)-\theta_{\mathrm{E}}$

$\mathrm{s}_{\mathrm{tI}}(\mathrm{k})=\mathrm{w}_{\mathrm{I}} \mathrm{s}_{\mathrm{I}}(\mathrm{k})-\mathrm{w}_{\mathrm{II}} \mathrm{u}_{\mathrm{I}}(\mathrm{k}-1)+\mathrm{w}_{\mathrm{EI}} \mathrm{u}_{\mathrm{E}}(\mathrm{k}-1)-\theta_{\mathrm{I}}$

이때 $\mathrm{w}_{\mathrm{E}}$ 와 $\mathrm{w}_{\mathrm{I}}$ 는 각각 여기와 금지 신경 단위의 비례상 수(scaling factor)이고, $\mathrm{w}_{\mathrm{EE}}$ 와 $\mathrm{w}_{\mathrm{II}}$ 는 자기 시냅스 연결 강 도를, $\mathrm{w}_{\mathrm{IE}}$ 와 $\mathrm{w}_{\mathrm{EI}}$ 는 상호 뉴런 시냅스 연결 강도, $\theta_{\mathrm{E}}$ 와 $\theta_{\mathrm{I}}$ 는 여기 및 금지 뉴런의 임계치을 각각 나타낸다. 뉴런의 여기와 금지 그룹의 활동은 다음 식에 의해 전개된다. 이 때 $\mathrm{r}_{\mathrm{E}}$ 와 $\mathrm{r}_{\mathrm{I}}$ 는 각각 여기와 금지 뉴런의재 점화할 수 없는 기간를 나타낸다.

$$
\begin{aligned}
& \mathrm{u}_{\mathrm{E}}(\mathrm{k}+1)=\mathrm{u}_{\mathrm{E}}(\mathrm{k})+\left(1-\mathrm{r}_{\mathrm{E}} \mathrm{u}_{\mathrm{E}}(\mathrm{k})\right) \Psi_{\mathrm{E}}\left[\mathrm{s}_{\mathrm{tE}}(\mathrm{k})\right] \\
& : \quad \text { 여기 뉴런 } \\
& \mathrm{u}_{\mathrm{I}}(\mathrm{k}+1)=\mathrm{u}_{\mathrm{I}}(\mathrm{k})+\left(1-\mathrm{r}_{\mathrm{I}} \mathrm{u}_{\mathrm{I}}(\mathrm{k})\right) \Psi_{\mathrm{I}}\left[\mathrm{s}_{\mathrm{tI}}(\mathrm{k})\right] \\
& : \quad \text { 금지 뉴런 }
\end{aligned}
$$

식 (8)과 (10)에 의해 등복각 곡선에 대한 방정식은 다 음과 같이 쓸 수 있다.

$$
\begin{aligned}
& \mathrm{u}_{\mathrm{I}}(\mathrm{k})=\frac{1}{\mathrm{w}_{\mathrm{IE}}}\left[\left(\mathrm{w}_{\mathrm{E}} \mathrm{s}_{\mathrm{E}}(\mathrm{k})-\Theta_{\mathrm{E}}\right.\right. \\
& \left.+\Psi_{\mathrm{E}}^{-1}\left[\frac{\mathrm{u}_{\mathrm{E}}(\mathrm{k})}{\left(1-\mathrm{r}_{\mathrm{E}} \mathrm{u}_{\mathrm{E}}(\mathrm{k})\right)}\right]+\mathrm{w}_{\mathrm{EE}} \mathrm{u}_{\mathrm{E}}(\mathrm{k})\right] \\
& \text { for } \mathrm{u}_{\mathrm{E}}(\mathrm{k}+1)=0 \\
& \mathrm{u}_{\mathrm{E}}(\mathrm{k})=\frac{1}{\mathrm{w}_{\mathrm{EI}}}\left[\left(-\mathrm{w}_{\mathrm{I}} \mathrm{s}_{\mathrm{I}}(\mathrm{k})-\Theta_{\mathrm{I}}\right.\right. \\
& \left.+\Psi_{\mathrm{I}}^{-1}\left[\frac{\mathrm{u}_{\mathrm{I}}(\mathrm{k})}{\left(1-\mathrm{r}_{\mathrm{I}} \mathrm{u}_{\mathrm{I}}(\mathrm{k})\right)}\right]+\mathrm{w}_{\mathrm{II}} \mathrm{u}_{\mathrm{I}}(\mathrm{k})\right] \\
& \text { for } \mathrm{u}_{\mathrm{I}}(\mathrm{k}+1)=0
\end{aligned}
$$

식 (11a)으로 정의되는 $\mathrm{u}_{I}$ 는 언제나 $\mathrm{u}_{\mathrm{E}}$ 의 단조 증가 함 수이다. 반면에, 식 (11b)에서 $\Psi_{\mathrm{I}}^{-1}$ 앞의 (-) 부호에 의해 $\mathrm{u}_{\mathrm{E}}$ 는 점차적으로 감소하는 $\mathrm{u}_{\mathrm{I}}$ 의 함수가 될 것이다. 두 등 복각 곡선 사이의 이러한 정성적인 차이는 여기와 금지 간의 반대칭에 대한 직접적인 결과이다. DNP의 응답 $\mathrm{u}(\mathrm{k})$ 는 신경 부차집단에서 여기와 금지의 개별적인 응답 인 $\mathrm{u}_{\lambda}(\mathrm{k})$ 의 중첩이며 다음으로 주어진다.

$$
\mathrm{u}(\mathrm{k})=\mathrm{u}_{\mathrm{E}}(\mathrm{k})+\mathrm{u}_{\mathrm{I}}(\mathrm{k})
$$

식 (12)의 의미는 신경 집단의 총체적인 활동이 여기 와 금지 후 시냅스 응답의 합이다.

\section{4. 자동화 시스템을 위한 학습 알고리듬}

학습 과정은 feedforward 및 feedback 가중치와 오차 함수를 최소화하기 위한 somatic gain의 적응과정을 포함 한다. 반복 학습 기법에서 제어의 순서는 각 반복 학습 단계에서 목표 상태 $\mathrm{u}_{\mathrm{d}}(\mathrm{k})$ 에 도달하기 위한 신경 출력 $\mathrm{u}(\mathrm{k})$ 를 발생하기 위해 변형된다. 학습의 반복 횟수가 증 가함에 따라 오차 $\mathrm{e}(\mathrm{k})$ 가 무한히 작은 값으로 줄어질 수 있다면 학습 방법은 수렴한다고 말한다. 즉, 초기조건의 임의 집합에 대해 오차 $\mathrm{e}(\mathrm{k})$ 와 매개변수 벡터 $\Omega\left(\mathrm{a}_{\mathrm{ff}}, \mathrm{b}_{\mathrm{ff}}, \mathrm{g}_{\mathrm{s}}, \mathrm{w}_{\mathrm{A}}\right)$ 의 성분은 각 학습 횟수 $\mathrm{k}$ 와 함께 변 한다.

$$
\begin{aligned}
& \mathrm{u}(\mathrm{k}) \rightarrow \mathrm{u}_{\mathrm{d}}(\mathrm{k}) \text { as } \mathrm{k} \rightarrow \infty \text { or, } \\
& \lim _{\mathrm{k} \rightarrow \infty}\left[\mathrm{u}_{\mathrm{d}}(\mathrm{k})-\mathrm{u}(\mathrm{k})=\mathrm{e}(\mathrm{k})\right] \rightarrow 0
\end{aligned}
$$

$\Omega\left(\mathrm{a}_{\mathrm{ff}}, \mathrm{b}_{\mathrm{ff}}, \mathrm{g}_{\mathrm{s}}, \mathrm{w}_{\lambda \wedge}\right)(\mathrm{k}+1)$ 을 구하기 위해서는 정보집 합 $\left\{\mathrm{e}(\mathrm{k}-\mathrm{m}), \mathrm{e}(\mathrm{k}), \Omega_{\left(\mathrm{a}_{\mathrm{ff}}, \mathrm{b}_{\mathrm{b}}, \mathrm{g}_{\mathrm{s}}, \mathrm{w}_{\mathrm{M}}\right)}(\mathrm{k})\right\}$ 만이 필요한데 이때 $\mathrm{m}=1,2, \cdots$ 이며 창의 크기를 결정한다. 학습 횟수가 증가 함에 따라 정보 집합은 다만 $\left\{\Omega_{\left(\mathrm{a}_{\mathrm{ff}}, \mathrm{b}_{\mathrm{f}}, \mathrm{g}_{\mathrm{s}}, \mathrm{w}_{\mathrm{k}}\right)}^{*}(\mathrm{k}), \mathrm{e}^{*}(\mathrm{k})\right\}$ 로 감소하며 이것은 $\mathrm{DNU}$ 매개변수와 오차가 최적치에 수렴 하였음을 가리킨다. 이를 위해 각 매개변수 벡터에 대해 최적화 되어야 하는 성능 지표는 다음과 같이 정의되며 이때 $\mathrm{E}$ 는 기대 연산자이다.

$$
\mathrm{J}=\mathrm{E}\left\{\mathrm{F}\left[\mathrm{e}\left(\mathrm{k} ; \Omega\left(\mathrm{a}_{\mathrm{ff}}, \mathrm{b}_{\mathrm{fb}}, \mathrm{g}_{\mathrm{s}}, \mathrm{w}_{\mathrm{AN}}\right)\right)\right]\right\}
$$

식 (14)에서 $\mathrm{F}\left[\mathrm{e}\left(\mathrm{k} ; \Omega\left(\mathrm{a}_{\mathrm{ff}}, \mathrm{b}_{\mathrm{fb}}, \mathrm{g}_{\mathrm{s}}, \mathrm{w}_{\mathrm{A}}\right)\right)\right]$ 의 일반적 인 형태는 오차의 우함수이다. 즉,

$$
\mathrm{J}=\frac{1}{2} \mathrm{E}\left\{\mathrm{e}^{2}\left(\mathrm{k} ; \Omega\left(\mathrm{a}_{\mathrm{ff}}, \mathrm{b}_{\mathrm{fb}}, \mathrm{g}_{\mathrm{s}}, \mathrm{w}_{\lambda \lambda}\right)\right)\right\}
$$

이때 $\mathrm{E}$ 는 기대 연산자이고 $\mathrm{e}(\mathrm{k})$ 는 목표 신호 $\mathrm{u}_{\mathrm{d}}(\mathrm{k})$ 와 실 제 신호 $\mathrm{u}(\mathrm{k})$ 간의 차이로 정의되는 오차 신호이다. 벡터 
$\Omega_{\left(a_{f f}, b_{f b}, g_{s}, w_{\Lambda \Lambda}\right)}$ 의 각 성분은 급경사 감소 (steepst-descent) 알고리듬에 의해 J를 최소화하는 방식으 로 적응되었다. 이 적응 알고리듬은 다음과 같이 쓸 수 있 는데 이때 $\Omega\left(\mathrm{a}_{\mathrm{ff}}, \mathrm{b}_{\mathrm{fb}}, \mathrm{g}_{\mathrm{s}}, \mathrm{w}_{\lambda \lambda}\right)(\mathrm{k}+1)$ 은 새로운 매개변수 벡터이고, $\Omega\left(\mathrm{a}_{\mathrm{ff}}, \mathrm{b}_{\mathrm{fb}}, \mathrm{g}_{\mathrm{s}}, \mathrm{w}_{\lambda \wedge}\right)(\mathrm{k})$ 는 현재 매개변수 벡터 이며 $\delta \Omega\left(\mathrm{a}_{\mathrm{ff}}, \mathrm{b}_{\mathrm{fb}}, \mathrm{g}_{\mathrm{s}}, \mathrm{w}_{\lambda \lambda}\right)(\mathrm{k})$ 는 매개변수 벡터의 적응 조절량이다. 급경사 감소법에서 매개변수 벡터의 조절은 성능지표 J에서 음의 기울기에 비례하도록 되어진다.

$$
\begin{aligned}
& \delta \Omega_{\left(\mathrm{a}_{\mathrm{ff}}, \mathrm{b}_{\mathrm{fb}}, \mathrm{g}_{\mathrm{s}}, \mathrm{w}_{\Lambda \lambda}\right)}(\mathrm{k}) \propto(-\nabla \mathrm{J}) \\
& \text { where, } \quad \nabla \mathrm{J}=\frac{\delta \mathrm{J}}{\delta \Omega_{\left(\mathrm{a}_{\mathrm{ff}}, \mathrm{b}_{\mathrm{fb}}, \mathrm{g}_{\mathrm{s}}, \mathrm{w}_{\mathrm{Ad}}\right)}}
\end{aligned}
$$

따라서, $\operatorname{dia}[\mu$ ]가 독립적인 적응 이득 행렬일 때 다음 과 같이 된다.

$$
\begin{aligned}
& \delta \Omega\left(\mathrm{a}_{\mathrm{ff}}, \mathrm{b}_{\mathrm{fb}}, \mathrm{g}_{\mathrm{s}}, \mathrm{w}_{\Lambda \lambda}\right) \\
& =-\operatorname{dia}[\mu] \frac{\delta \mathrm{J}}{\delta \Omega\left(\mathrm{a}_{\mathrm{ff}}, \mathrm{b}_{\mathrm{fb}}, \mathrm{g}_{\mathrm{s}}, \mathrm{w}_{\Lambda \Lambda}\right)} \\
& =-\operatorname{dia}[\mu] \nabla J
\end{aligned}
$$

위의 식에서 $\operatorname{dia}[\mu]$ 는

$\operatorname{dia}[\mu]=\left[\begin{array}{cccc}\mu_{a_{i}} & 0 & 0 & 0 \\ 0 & \mu_{b_{j}} & 0 & 0 \\ 0 & 0 & \mu_{g_{s}} & 0 \\ 0 & 0 & 0 & \mu_{\lambda \lambda}\end{array}\right]$

이때 $\mu_{a_{i}}, i=0,1,2, \mu_{b_{j}}, j=1,2, \mu_{g_{s}}$ 는 $\mathrm{DNU}$ 의 적응 매개변수의 독립적인 학습 이득이고, $\mu_{\lambda \lambda^{\prime}}$ 는 자 기 및 상호 뉴런 시냅스 연결의 학습 이득을 나타낸다. $\Phi_{\left(\mathrm{a}_{\mathrm{ff}}, \mathrm{b}_{\mathrm{fb}}\right)}$ 로 $\mathrm{DNU}$ 의 시냅스 가중치 벡터를 나타내면 $\Phi_{\left(\mathrm{a}_{\mathrm{ff}}, \mathrm{b}_{\mathrm{fb}}\right)}$ 에 대한 성능 지표의 기울기는 식 (19)으로 구 해진다.

$$
\mathrm{P} \Phi_{\left(\mathrm{a}_{\mathrm{ff}}, \mathrm{b}_{\mathrm{fb}}\right)}(\mathrm{k})=\frac{\delta_{\mathrm{V}}(\mathrm{k})}{\delta \Phi_{\left(\mathrm{a}_{\mathrm{ff}}, \mathrm{b}_{\mathrm{fb}}\right)}}=\mathrm{g}_{\mathrm{s}} \frac{\delta_{\mathrm{V}_{1}}(\mathrm{k})}{\delta \Phi_{\left(\mathrm{a}_{\mathrm{ff}}, \mathrm{b}_{\mathrm{fb}}\right)}}
$$

는 매개변수-상태(또는 감도) 신호의 벡터를 나타낸다. feedforward와 feedback 가중치에 대한 매개변수 상태 신 호는 각각 식 (20)의 관계로 주어진다.

$$
\begin{aligned}
& \frac{\delta \mathrm{J}}{\delta \phi_{\left(\mathrm{a}_{\mathrm{ff}}, \mathrm{b}_{\mathrm{b}}\right)}} \\
= & \frac{1}{2} \mathrm{E}\left[\frac{\delta\left[\mathrm{u}_{\mathrm{d}}(\mathrm{k})-\mathrm{u}(\mathrm{k})\right]^{2}}{\delta \phi_{\left(\mathrm{a}_{\mathrm{ff}}, \mathrm{b}_{\mathrm{b}}\right)}}\right] \\
= & \mathrm{E}\left[\mathrm{e}(\mathrm{k})\left\{-\frac{\delta \Psi(\mathrm{v})}{\delta \phi_{\left(\mathrm{a}_{\mathrm{ff}}, \mathrm{b}_{\mathrm{rb}}\right)}}\right\}\right] \\
= & \mathrm{E}\left[-\mathrm{e}(\mathrm{k})\left\{\frac{\delta \Psi(\mathrm{v})}{\delta \mathrm{v}} \frac{\delta \mathrm{v}}{\delta \phi_{\left(\mathrm{a}_{\mathrm{ff}}, \mathrm{b}_{\mathrm{b}}\right)}}\right\}\right] \\
= & \mathrm{E}\left[-\mathrm{e}(\mathrm{k})\left\{\operatorname{sech}^{2}[v(k)] P \phi_{\left(a_{f f}, b_{f b}\right)}\right\}\right] \\
& \mathrm{P} \Phi_{\left(\mathrm{a}_{\mathrm{ff}} \mathrm{f}\right.}(\mathrm{k})=\mathrm{g}_{\mathrm{s}}[\mathrm{s}(\mathrm{k}-\mathrm{i})], \mathrm{i}=0,1,2, \\
& \mathrm{P} \Phi_{\mathrm{b}_{\mathrm{ff}},}(\mathrm{k})=-\mathrm{g}_{\mathrm{s}}\left[\mathrm{v}_{1}(\mathrm{k}-\mathrm{j})\right], \mathrm{j}=1,2
\end{aligned}
$$

somatic gain gs에 대한 성능지표의 기울기는 다음으 로 주어진다.

$$
\begin{aligned}
\frac{\delta \mathrm{J}}{\delta \mathrm{g}_{\mathrm{s}}} & =\frac{1}{2} \mathrm{E}\left[\frac{\delta\left[\mathrm{u}_{\mathrm{d}}(\mathrm{k})-\mathrm{u}(\mathrm{k})\right]^{2}}{\delta \mathrm{g}_{\mathrm{s}}}\right] \\
& =\mathrm{E}\left[-\mathrm{e}(\mathrm{k})\left\{\operatorname{sech}^{2}[v(k)] v_{1}(k)\right\}\right]
\end{aligned}
$$

자기 및 상호 뉴런 시냅스 연결에서의 적응은 다음과 같이 구할 수 있다.

$$
\begin{aligned}
& \frac{\delta \mathrm{J}}{\delta \mathrm{w}_{\lambda \lambda^{\prime}}}=\frac{1}{2} \mathrm{E}\left[\frac{\delta\left[\mathrm{u}_{\mathrm{d}}(\mathrm{k})-\mathrm{u}(\mathrm{k})\right]^{2}}{\delta \mathrm{w}_{\lambda \lambda^{\prime}}}\right] \\
& =\mathrm{E}\left[-\mathrm{e}(\mathrm{k})\left\{\frac{\delta \Psi(\mathrm{v})}{\delta \mathrm{v}} \frac{\delta \mathrm{v}}{\delta \mathrm{w}_{\lambda \lambda^{\prime}}}\right\}\right] \\
& =\mathrm{E}\left[-\mathrm{e}(\mathrm{k})\left\{\operatorname{sech}^{2}[v(k)] g_{s} u_{\lambda}(k-1)\right\}\right]
\end{aligned}
$$

위의 식들로부터 DNP 매개변수 갱신 알고리듬은 다 음과 같이 쓸 수 있다[8-10].

$$
\begin{aligned}
& \mathrm{a}_{\mathrm{ff}_{\mathrm{i}}}(\mathrm{k}+1)=\mathrm{a}_{\mathrm{ff}_{\mathrm{i}}}(\mathrm{k}) \\
& +\mu_{\mathrm{a}_{\mathrm{i}}} \mathrm{E}\left[\mathrm{e}(\mathrm{k}) \operatorname{sech}^{2}[\mathrm{v}(\mathrm{k})] \mathrm{P} \phi_{\mathrm{aff}_{\mathrm{f}}}(\mathrm{k})\right], \\
& \mathrm{i}=0,1,2
\end{aligned}
$$

$$
\mathrm{b}_{\mathrm{fb}_{\mathrm{j}}}(\mathrm{k}+1)=\mathrm{b}_{\mathrm{fb}_{\mathrm{j}}}(\mathrm{k})
$$$$
+\mu_{b_{j}} E\left[e(k) \operatorname{sech}^{2}[\mathrm{v}(\mathrm{k})] \mathrm{P} \Phi_{\mathrm{b}_{\mathrm{fb}},}(\mathrm{k})\right] \text {, }
$$$$
\mathrm{j}=1,2
$$

$$
\begin{aligned}
& \mathrm{g}_{\mathrm{s}}(\mathrm{k}+1)=\mathrm{g}_{\mathrm{s}}(\mathrm{k}) \\
& \quad+\mu_{\mathrm{g}_{\mathrm{s}}} \mathrm{E}\left[\mathrm{e}(\mathrm{k}) \operatorname{sech}^{2}[\mathrm{v}(\mathrm{k})] \mathrm{v}_{1}(\mathrm{k})\right]
\end{aligned}
$$

$\mathrm{w}_{\lambda \lambda^{\prime}}(\mathrm{k}+1)=\mathrm{w}_{\lambda \lambda^{\prime}}(\mathrm{k})+\mu_{\lambda \lambda^{\prime}} \cdot \mathrm{E}$

$\left[-\mathrm{e}(\mathrm{k})\left\{\operatorname{sech}^{2}[v(k)] g_{s} u_{\lambda}(k-1)\right\}\right]$ 
식(23a)과 (23b)는 시냅스 가중치에서, (23c)는 DNU의 sigmoidal gain에서, 식 (23d)은 외부 시냅스 가중치에서 적응성을 갖는다.

\section{5. 자동화 시스템의 온 라인 학습}

\section{1 학습 기법}

그림 3과 같이 제안된 학습 기법은 주어진 목표 직각 좌표의 집합에 대한 관절각을 결정하기 위해 $\mathrm{DNP}$ 를 사 용한다. 오차 신호는 목표 위치와 관측 위치의 차로 계산 된다. 신경 가중치는 다음절에서 개발되는 학습 알고리듬 을 바탕으로 적응된다. 제안된 신경 프로세서와 학습 기 법의 효과를 보이기 위해 본 논문에서는 인간 다리의 모 델로서 2 링크 구조가 고려된다. 어떤 범위 내에서 일어 나는 회전 운동에서의 관절은 인간 다리의 엉덩이와 무 릎 관절과 유사하다. 종점이라고도 부르는 두 번째 링크 의 자유로운 팁은 직각 좌표 시스템에 기초한 궤도를 나 타낸다. 좌표계의 원점은 공간상에 고정되어 있다고 가정 하는 첫번째 (엉덩이) 관절이고 반면 종점 좌표 $(\mathrm{x}, \mathrm{y})$ 는 두 수직축 $\mathrm{X}, \mathrm{Y}$ 에 대해 위치한다. 둔부 관절은 정점으로 간주된다. 다리의 위치는 또한 그림과 같이 기준을 따라 두 관절이 형성하는 각에 의해 제한될 수 있다. 이들 두 각의 관계는 $\theta_{1}$ 과 $\theta_{2}$ 로 정의되며 종점 좌표 $\mathrm{x}, \mathrm{y}$ 는 다음 과 같은 2 링크 다리의 역학 방정식을 형성한다.

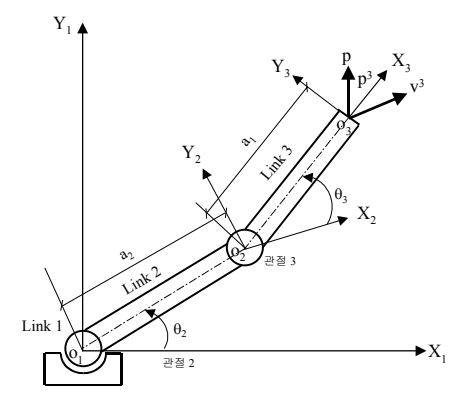

[그림 3] 2축 매니퓰레이터의 구조

$$
\begin{aligned}
& \mathrm{x}=\mathrm{L}_{1} \cos \left(\Theta_{1}\right)+\mathrm{L}_{2} \cos \left(\Theta_{1}+\Theta_{2}\right) \\
& \mathrm{y}=\mathrm{L}_{1} \sin \left(\Theta_{1}\right)+\mathrm{L}_{2} \cos \left(\Theta_{1}+\Theta_{2}\right)
\end{aligned}
$$

자동화 시스템의 역기구학적인 문제의 한가지 해법은 다 중 해의 발생을 피할 수 있는 어떤 편리한 각의 범위로 두 링크의 운동을 제한하는 것이다. 부수적으로 무릅 관절과 같이 경첩 구조를 가진 인간 다리의 구조는 정강이의 제한
된 움직임을 허용한다. 위에서 언급한 제약들에 대해 생각 해 보면 2 링크 다리 모델의 두 관절은 특별한 각 범위 $\left(-30^{\circ}<\theta{ }_{1}<180^{\circ}, 0^{\circ}<\theta{ }_{2}<180^{\circ}\right)$ 내에서 움직이도록 제한된다.

\section{2 컴퓨터 모의 실험}

만약 요구되는 출력이 $y_{t}(k)$ 이고 제어중인 플랜트의 실제 출력이 $y(k)$ 일때 오차가 $e(k)=y_{d}(k)-y(k)$ 이 면 제어의 목표는 다음과 같은 제한된 제어 입력 $u(k)$ 를 결정하는 것이다.

$$
\lim _{k \rightarrow \infty}\left[y_{d}(k)-y(k)\right]=e(k)=0
$$

제어되는 플랜트는 다음 차분 방정식으로 기술된다.

$\mathrm{y}(\mathrm{k})=\mathrm{f}[\mathrm{y}(\mathrm{k}-1), \mathrm{y}(\mathrm{k}-2), \mathrm{u}(\mathrm{k}), \mathrm{u}(\mathrm{k}-1), \mathrm{u}(\mathrm{k}-2)]$

예 1 : 이 예에서는 end-effector의 좌표 $\mathrm{x}$ 와 $\mathrm{y}$ 가 임의 로 선택되어 DNP의 입력으로 인가되었다. 신경 가중치 는 출력 오차가 미리 정의된 값 0.05 로 감소될 때까지 조 절된다. 그림 4는 2 링크 매니퓰레이터의 실제 및 학습된 좌표를 보이고 있다.

예 2 : 매니퓰레이터의 성공적인 동작은 구조상에 동 적인 변화를 초래할 수도 있는 섭동에 대한 대처 능력에 달려 있다. 이 예에서는 링크 $\mathrm{L}_{2}$ 가 개선된 효과를 보인다. 이러한 동적 섭동으로 인하여 관찰된 end-effector의 위치 는 신경망 가중치의 재 조절을 요하는 목표 위치와 일치 해야 한다. 따라서 그림 5 의 결과로부터 DNP가 자동화 시스템을 위한 매니퓰레이터의 역학적 변화에 적응할 수 있음을 보이며 따라서 학습 기법의 강인성을 보인다.

\section{6. 결론}

본 연구에서는 자동화 시스템의 비선형 매개변수의 강 인한 동특성이 중시되는 $\mathrm{DNP}$ 신경망 구조를 제안하였 다. 이 방법은 목표 직각 좌표를 입력으로 받아들여서 매 니퓰레이터의 관절각을 결정할 수 있었다. 따라서 비선형 동적 매니퓰레이터의 관절각을 정하여진 작업 영역내에 서 보다 빠르고 정확하게 운동할 수 있었으며, 또한 기존 신경망 제어기보다 학습 기법의 강인성과 적응성을 동적 매니퓰레이터에서 확인하였다. 앞으로의 과제는 본 논문 에서 개발한 유연한 구조와 복잡한 자동화 시스템에 대 한 확장이 요구된다. 


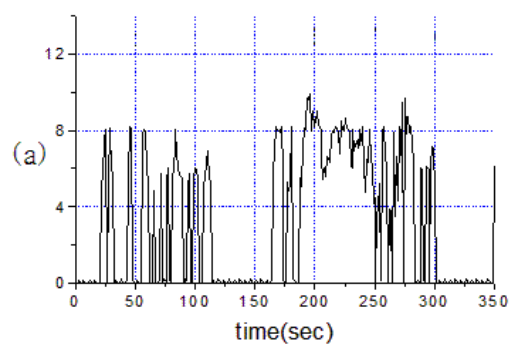

+ : 기준궤적, * : 출력궤적

(a) DNU 제어기에 의해 학습된 2 링크 매니퓰레이터의 궤적 좌표

(b)

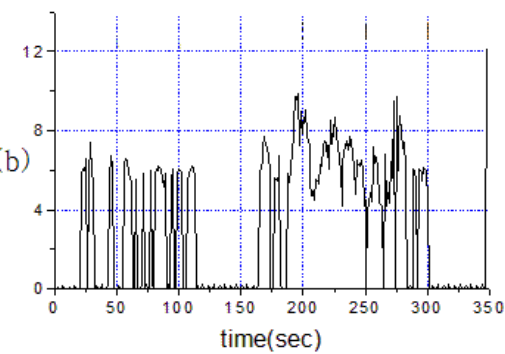

+ : 기준궤적, * : 출력궤적

(b) DNP 제어기에 의해 학습된 2링크 매니퓰레이터의 궤 적 좌표

[그림 4] 예 2; DNP 제어기에 의해 학습된 2링크 매니퓰 레이터의 궤적 좌표
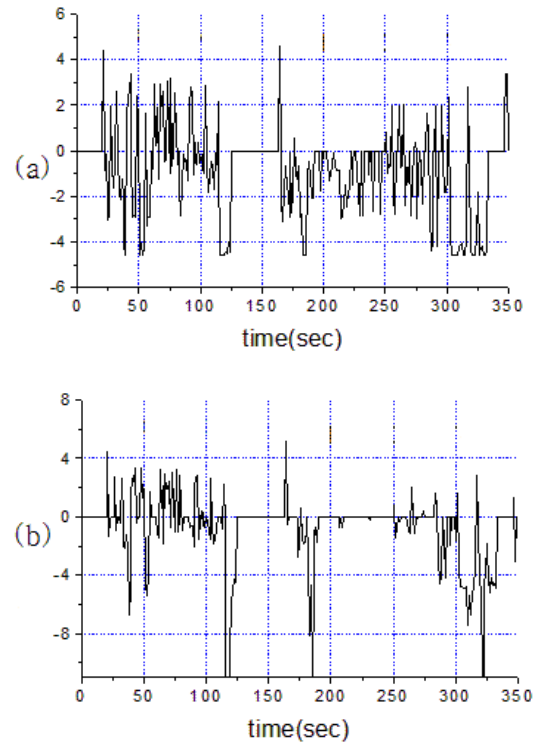

[그림 5] 예 $2 ; \mathrm{K}=100$ 에서 $\mathrm{L}_{2}$ 관절의 길이가 0.5-0.7로 변화 시 매니퓰레이터 관절의 오차 궤적

\section{참고문헌}

[1]. M.M.Gupta, D.H.Rao, Dynamic Neural Units with Applications to the Control of Unknown Linear System, in Journal of Intelligent and Fuzzy Systems, pp.73-92, vol.1, no.1

[2] Alen Varsek, Tanja Urbancic, Bodgan Filipic, Genetic Algorithms in Controller Design and Tunning, IEEE Trans. On Systems, Man, and Cybernetics, vol.23, no.5, pp.1330-1339, 2007.

[3]. J-J. E. slotine and W. Li, Applied Nonlinear control, Prentice-Hall. 2006.

[4]. J-J. E. slotine, "Sliding controller Design for Non-linear system", Int. J. control, Vol. 40, No. 2, pp. $421 \sim 434,2005$.

[5]. K. S. Narenda, K. Parthasarathy, "Identification and control of dynamical system using neural networks", IEEE Trans. on Neural Network, Vol. 1, No.1, pp. $4 \sim 27$, March 2007.

[6]. F. C. Chen, "Back-propagation neural networks for nonlinear self-tuning adaptive control”, IEEE control systems Magazine, pp. 44-48, April 2008.

조 현 섭(Hyun-Seob Cho)

[종신회원]

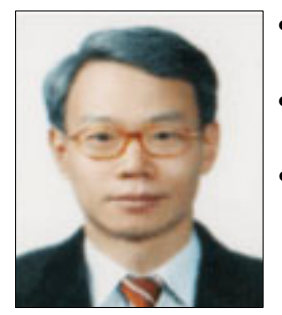

- 1990년 2월 : 원광대학교 공과대 학 전기공학과 졸업

- 1992년 2월 : 원광대학교 공과대 학 전기공학과 (석사)

- 1996년 2월 : 원광대학교 공과대 학 전기공학과 (박사)

- 1996년 1월 1997년 6월 : Department of Electrical and Computer Engineering, University of California Irvine(UCI) 연구원

- 1998년 1월 현재 : 한국전력기술인협회 고급감리원 (전력감리)

- 1997년 3월 현재 : 청운대학교 부교수

<관심분야>

전기공학, 공장자동화, 응용전자 